\title{
Measuring short-term and long-term physiological stress effects by cortisol reactivity in saliva and hair
}

\author{
Berry J. van Holland • Monique H. W. Frings-Dresen • \\ Judith K. Sluiter
}

Received: 8 July 2011 / Accepted: 1 December 2011 / Published online: 20 December 2011

(C) The Author(s) 2011. This article is published with open access at Springerlink.com

\begin{abstract}
Purpose The aims of this study were to investigate (1) the concurrent relationship between short-term and long-term stress reactivity measured by cortisol excretion and (2) the relationship of these physiological stress effects with selfreported stress and need for recovery after work (NFR).

Methods Participants were production workers in the meat-processing industry. Short-term cortisol excretion was calculated by summing 18 saliva samples, sampled over a 3-day period. Samples were delivered by 37 participants. Twenty-nine of them also supplied one hair sample of at least $3 \mathrm{~cm}$ in length for an analysis of long-term (3 months) cortisol excretion. All of them filled in a short questionnaire on self-reported stress and NFR. Self-reported stress was assessed by a three-item stress screener; NFR was assessed by an 11-item scale.

Results Short-term and long-term cortisol excretion are significantly, but moderately, associated $(r=0.41, P=0.03)$. Short-term and long-term cortisol excretion correlated weakly to self-reported stress and NFR (correlations varied from -0.04 to 0.21 ).

Conclusions Short-term and long-term physiological stress excretion levels are moderately associated. Physiological stress effects assessed from saliva and hair cannot be used interchangeably with self-reported stress because they only correlate weakly. To better predict long-term cortisol excretion in workers, the predictive value of short-term cortisol excretion must be evaluated in a prognostic longitudinal study in a working population.
\end{abstract}

B. J. van Holland · M. H. W. Frings-Dresen · J. K. Sluiter $(\square)$ Coronel Institute of Occupational Health, Academic Medical Center, University of Amsterdam, PO Box 22700,

1100 DE Amsterdam, The Netherlands

e-mail: j.sluiter@amc.uva.nl
Keywords Hair $\cdot$ Saliva $\cdot$ Cortisol $\cdot$ Stress $\cdot$ Measurement

\section{Introduction}

Over the past decade, stress has received increasing attention, particularly in relation to stress factors experienced by workers, self-reported stress and objective measurements of stress (Chida and Steptoe 2009; Maina et al. 2008; Sluiter et al. 1998). Research into stress hormone reactivity is quite common, especially when measured in urine, blood and saliva (Maina et al. 2008; Sluiter et al. 1998; Evolahti et al. 2006). These body fluids are used to measure short-term cortisol excretion. The relationship between short-term salivary cortisol excretion and self-reported psychological stress has frequently been investigated. However, results of these studies show different outcomes. Dettenborn et al. (2010) stated that a lack of an association between these parameters is not uncommon in the literature.

However, few studies have examined long-term physiological stress effects because of a lack of biomarkers. Recently, a new procedure has been developed to measure cumulative stress hormone reactivity, that is, cortisol, in human hair. Long-term cortisol excretion can now be accurately measured, up to 6 months back (Dettenborn et al. 2010). Sauvé et al. (2007) reported a significant, but moderate, correlation $(r=0.33, P=0.04)$ between 24-h urinary cortisol excretion and hair cortisol concentrations in humans. Only one study reported measuring both long-term (in hair) and short-term (in saliva) cortisol excretion simultaneously in a mixed group of anxious and non-anxious subjects (Steudte et al. 2010). No significant correlations $(r=0.27)$ were found in that study, perhaps due to the fact that too few saliva measurements were incorporated ( 2 days, 6 samples/day) or the mean value that was calculated. 
Davenport et al. (2006) did find a significant correlation between hair and salivary cortisol reactivity in rhesus macaque monkeys, but they point out that this relationship has to be investigated for any new species being tested.

To study whether short-term cortisol excretion can predict long-term cortisol excretion, it seemed plausible to first study their concurrent relationship. If the concurrent relationship between current salivary cortisol excretion and retrospective excretion in hair is strong enough, it is necessary to set up a longitudinal study to investigate the predictive value of short-term cortisol excretion on long-term cortisol excretion in a criterion-related validity study.

To gain a further understanding of acute and chronic stress reactivity and their relationship, we set out to investigate these parameters in a working population. The aim was to investigate the concurrent association between short-term and long-term cortisol reactivity. We also investigated how self-reported stress is associated with physiological cortisol reactivity in saliva and hair.

\section{Methods}

Participants were recruited from companies in the Dutch meat-processing industry as part of a larger workload study. Forty-two production workers were approached from eight organizations that were appointed for this study by a committee of employers and employees of the meat-procession sector to participate in this study. Participants received oral and written instructions about the protocol. Participation was voluntary. After signing the informed consent form, measurements were initiated.

Participation consisted of collecting saliva samples on 3 days, that is, two working days and one day off, within 7 days. Each participant received 6 Salivettes (Sarstedt, Etten-Leur, The Netherlands) per day and was instructed to take a sample at prescribed times (9:00 a.m., 11:00 a.m., 1:00 p.m., 3:00 p.m., 5:00 p.m., 8:00 p.m.). The exact time of sample collection was noted, next to possible peculiarities. Peculiarities were, for instance, events that could disturb cortisol production.

A single strand of hair of at least $3 \mathrm{~cm}$ in length was cut from the back of the skull. One centimetre of hair represents the accumulation effects of stress for approximately 1 month (Gow et al. 2010). In this way, cumulative stress reactivity of the past 3 months could be determined.

Self-reported stress effects were assessed by the validated stress screener (Braam et al. 2009) and recovery problems after working time. The need for recovery after work was assessed by an 11-item instrument as described by De Croon et al. (2003). Participants filled in the questionnaire at the same time as the hair samples were collected.
Saliva and hair analyses were performed at the laboratory of Prof. Dr. C. Kirschbaum in Dresden, Germany. The protocol for saliva analysis is described by Strahler et al. (2010), and the protocol for hair analysis by Kirschbaum et al. (2009).

Participants without salivary cortisol data were excluded from the analyses. For the remaining data, missing individual salivary cortisol values were replaced by group means of the specific time of day. For the analyses, all salivary cortisol concentrations within subjects were summed to calculate an accumulated short-term stress marker over a 3 -day period. For the stress screener $(\min 0-\max 6)$ and NFR ( $\min 0-\max 100$ ), scale scores were calculated. Pearson's correlation coefficient (r) was calculated between short-term and long-term cortisol excretion, and $R^{2}$ was calculated from there. Cohen's criteria (Cohen 1998) for correlations were used: low when $r=0.1-0.3$, moderate when $r=0.3-0.5$, and high when $r=0.5-1.0$. Furthermore, Pearson's correlations were calculated between short-and longterm cortisol excretion, self-reported stress, and NFR. For all analyses, the significance level was set at $P<0.05$. Results are presented as means $( \pm \mathrm{SD})$.

\section{Results}

Useful saliva measurements were collected from 37 workers, and useful hair measurements were collected from 29 workers. Complete data were available from 27 participants. Among the participants, $81 \%$ were men and $19 \%$ were women. The average age of the participants was 46 $( \pm 10)$ years, and their average body mass index (BMI) was $26( \pm 4) \mathrm{kg} / \mathrm{m}^{2}$.

Short-term cortisol excretion was on average (SD) 114.2 $( \pm 38.5) \mathrm{nmol} / \mathrm{l}$. Long-term cortisol excretion was on average (SD) $15.4( \pm 8.7) \mathrm{pg} / \mathrm{mg}$.

Correlations are displayed in Table 1. Short-term and long-term cortisol excretion correlated significantly and moderately $(r=0.41, P=0.03)$. The variation in short-term

Table 1 Correlations between need for recovery after work, stress complaints, short-term physiological stress effects and long-term physiological stress effects

\begin{tabular}{clll}
\hline & $\begin{array}{l}\text { Short-term } \\
\text { cortisol excretion }\end{array}$ & $\begin{array}{l}\text { Stress } \\
\text { complaints }\end{array}$ & $\begin{array}{l}\text { Need for } \\
\text { recovery }\end{array}$ \\
\hline Long-term & $r=0.41$ & $r=0.12$ & $r=0.08$ \\
cortisol excretion & $P=0.03^{*}$ & $P=0.54$ & $\begin{array}{l}P=0.70 \\
n=29\end{array}$ \\
& $n=29$ & $n=28$ & $n=-0.04$ \\
Short-term & & $r=0.21$ \\
cortisol excretion & & $P=0.81$ & $P=0.22$ \\
& & $n=36$ & $n=37$ \\
\hline
\end{tabular}

$* P<0.05$ 
cortisol excretion explains about $17 \%$ of the variance in long-term cortisol excretion $\left(R^{2}=0.17\right)$.

Correlations between stress complaints or need for recovery and physiological stress reactivity were low and varied between -0.04 and 0.21 .

\section{Discussion}

Short-term and long-term cortisol reactivity representing short-term and long-term physiological stress levels are moderately associated. Physiological stress levels assessed from saliva and hair cannot be used interchangeably with self-reported stress in this working population because they correlate only weakly.

This paper presents unique material on measurement of short-term and long-term physiological stress reactivity in one group of workers. Both short-term and long-term cortisol reactivity have been investigated within subjects to elucidate their relationship. Also, short-term stress reactivity has been represented as an accumulation of multiple acute cortisol measurements over a time period of 3 days, which has not been presented before.

The hair cortisol levels are comparable to those reported by Dettenborn et al. (2010) and Steudte et al. (2010). Shortterm cortisol excretion has not been presented in a similar way, but individual cortisol values were comparable to those reported by Steudte et al. (2010) and Strahler et al. (2010).

Short-term and long-term cortisol reactivity correlate moderately. This leads to the suggestion that acute stress effects may, in the long run, lead to chronic stress effects. These results are supported by the findings of Sauvé et al. (2007), who reported the same correlation $(r=0.33$, $P=0.04$ ) between 24-h (acute) urinary cortisol concentrations and hair cortisol. They also reported a non-significant correlation between hair cortisol and salivary cortisol ( $r=0.31, P=0.12$ ), but in that study, only 1 saliva sample was obtained between 7:30 and 10:00 a.m..

Self-reported stress included both past and present experiences. Participants were asked about their experiences over the past weeks in the self-reports. No significant correlation was found between short- or long-term cortisol excretion and self-reported stress levels. Therefore, cortisol excretion and self-reported stress do not represent the same concept. Another explanation might be the timeline, that is, retrospective assessment of self-reported stress levels of several days or weeks, prospective short-term cortisol excretion (today and for two more days in the coming week), and retrospective estimate of long-term cortisol excretion (representing the last 3 months), and would suggest change to the planning of reports and sampling in future studies.
Need for recovery after work showed low associations with the parameters of physiological stress effects in this study. Possible explanations for these findings might be the fact that we averaged working days with days off. However, in earlier studies, both urinary cortisol values of only working days and days off correlated with need for recovery (Sluiter et al. 2001. Another explanation could be that only part of the evening (up to a 2000 hours sample) was included in this study, and therefore, only part of the recovery time after work was included. An explanation for the low association findings of need for recovery levels with the long-term cortisol excretion might be the reversed timescale: need for recovery after working time is evaluated during the last 2 weeks while the physiological parameter in hair mirrored the three last months. More studies would be necessary to gain knowledge on this topic.

Because our saliva samples were only measured during daytime (9 a.m. till 8 p.m.) while hair cortisol would theoretically be dependent on both day and night time, one could argue that a more ideal design would have included evening and night samples as well. As this was not the case, we could acknowledge this as a limitation of our study. However, we are not so worried about this issue because in earlier studies that we performed while using urinary cortisol sampling throughout day and night, we did not find large differences in mean excretion rate between night and morning time periods (Sluiter et al. 2000b).

For a long time, cortisol reactivity could only be measured in a way that would represent the short-term stress hormone reactivity in blood, urine or saliva, but the development of hair analysis has provided new opportunities. By using hair cortisol, opportunities for cumulated stress reactivity over a much longer period of time are possible and can be measured as long-term indicators of stress reactivity. A benefit in comparison with urine, saliva or blood collection (Sluiter et al. 2000) is that this method is less elaborate as well.

It can be concluded that short-term stress hormone reactivity is moderately associated with long-term stress reactivity when both are assessed concurrently. Self-reported parameters of stress estimated over a few weeks were not valuable in this study to predict short-term and long-term cortisol excretion. Therefore, to measure self-reported stress levels, questionnaires are still the preferred assessment method.

Ideally, when short-term cortisol reactivity is used to predict future long-term reactivity, the order of sampling should be reversed compared to what was done in this study. It is recommended that a longitudinal study be conducted to answer our question in a predictive way. 
Acknowledgments This study was supported by a grant from the Collective Labour Agreement Parties of the Meat Processing Industry in The Netherlands. We are grateful to the workers who agreed to participate in this part of the research project.

Conflict of interest The authors declare that they have no conflict of interest.

Open Access This article is distributed under the terms of the Creative Commons Attribution Noncommercial License which permits any noncommercial use, distribution, and reproduction in any medium, provided the original author(s) and source are credited.

\section{References}

Braam C, Van Oostrom SH, Terluin B, Vasse R, De Vet HCW, Anema JR (2009) Validation study of a distress screener. J Occup Rehabil 19:231-237. doi:10.1007/s10926-009-9178-z

Chida Y, Steptoe A (2009) Cortisol awakening response and psychosocial factors: a systematic review and meta-analysis. Biol Psychol 80:265-278. doi:10.1016/j.bio[sycho.2008.10.004

Cohen J (1998) Statistical power analysis for the behavioral sciences, 2nd edn. Lawrence Erlbaum Associates, Hillsdale, NJ

Davenport MD, Tiefenbacher S, Lutz CK, Novak MA, Meyer JS (2006) Analysis of endogenous cortisol concentrations in the hair of rhesus macaque monkeys. Gen Comp Endocrinol 147:255261. doi:10.1016/j.ygcen.2006.01.005

De Croon EM, Sluiter JK, Frings-Dresen MHW (2003) Psychometric properties of the need for recovery after work scale: test-retest reliability and sensitivity to detect change. Occup Environ Med 63:202-206. doi:10.1136/oem.2004.018275

Dettenborn L, Tietze A, Bruckner F, Kirschbaum C (2010) Higher cortisol content in hair among long-term unemployed individuals compared to controls. Psychoneuroendocrinology 35:1404-1409. doi:10.1016/j.psyneuen.2010.04.006

Evolahti A, Hultcrantz M, Collins A (2006) Women's work stress and cortisol levels: a longitudinal study of the association between the psychosocial work environment and serum cortisol. J Psychosom Res 61:645-652. doi:10.1016/j.jpsychores.2006.07.022
Gow R, Thomson S, Rieder M, Van Uum S, Koren G (2010) An assessment of cortisol analysis in hair and its clinical applications. Forensic Sci Int 196:32-37. doi:10.1016/j.forsciint.2009.12.040

Kirschbaum C, Tietze A, Skoluda N, Dettenborn L (2009) Hair as a retrospective calendar of cortisol production-increased cortisol incorporation into hair in the third trimester of pregnancy. Psychoneuroendocrinology 34:32-37. doi:10.1016/j.psyneuen. 2008.08.024

Maina G, Palmas A, Filon FL (2008) Relationship between self-reported mental stressors at the workplace and salivary cortisol. Int Arch Occup Environ Health 81:391-400. doi:10.1007/s00420007-0224-X

Sauvé B, Koren G, Walsh G, Tokmakejian S, Van Uum SHM (2007) Measurement of cortisol in human hair as a biomarker of systemic exposure. Clin Invest Med 30(5):E183-E191

Sluiter JK, Van der Beek AJ, Frings-Dresen MHW (1998) Work stress and recovery measured by urinary catecholamines and cortisol excretion in long distance coach drivers. Occup Environ Med 55:407-413

Sluiter JK, Frings-Dresen MH, Meijman TF, van der Beek AJ (2000a) Reactivity and recovery from different types of work measured by catecholamines and cortisol: a systematic literature overview. Occup Environ Med 57:298-315

Sluiter JK, Frings-Dresen MHW, vander Beek AJ, Meijman TF, Heisterkamp SJ (2000b) Neuroendocrine reactivity and recovery from work with different physical and mental demands. Scand J Work Environ Health 26(4):306-316

Sluiter JK, Frings-Dresen MHW, vander Beek AJ, Meijman TF (2001) The relation between work-induced neuroendocrine reactivity and recovery, subjective need for recovery, and health status. J Psychosom Res 50:29-37

Steudte S, Stalder T, Dettenborn L, Klumbies E, Foley P, BeesdoBaum K, Kirschbaum C (2010) Decreased hair cortisol concentrations in general anxiety disorder. Psychiatr Res 186:310-314. doi:10.1016/k.psychres.2010.09.002

Strahler J, Berndt C, Kirschbaum C, Rohleder N (2010) Aging diurnal rhythms and chronic stress: distinct alteration of diurnal rhythmicity of salivary $\alpha$-amylase and cortisol. Biol Psychol 84:248256. doi:10.1016/j.biopsycho.2010.01.019 